\title{
Some effects of 1 week's monocular exposure to long-wavelength stimuli
}

\author{
ALVIN EISNER and JAY M. ENOCH \\ School of Optometry, University of California, Berkeley, California 94720
}

\begin{abstract}
Subjects wore a long-wavelength passband filter over one eye for 1 week. As a consequence, for that eye only, sensitivity to long-wavelength stimuli declined, unique yellow shifted to longer wavelengths, and scotopic stimuli acquired a strikingly bluish appearance. These results make it very likely that long-term exposure to a long-wavelength world can induce relatively prolonged (at least hours) postreceptoral adaptation.
\end{abstract}

There have been many studies of the visual system's response to restricted environments or to conditions of deprivation. Typically, these studies have been conducted with infant animals. For example, kittens have been reared in environments lacking horizontal or vertical contours (Blakemore \& Cooper, 1970; Hirsch \& Spinelli, 1970) and infant monkeys have been rendered strabismic, thus precluding any functional binocular vision (Baker, Griff, \& von Noorden, 1974; Wiesel \& Hubel, 1974). The general finding is that lack of experience with some relevant features in the visual environment leads to a greatly reduced response to these stimuli, both physiologically and behaviorally, when they are presented to the animal later in its life (Barlow, 1975; Kuffler \& Nichols, 1976, chap. 19). This is true for human vision under naturally occurring conditions of sensory deprivation as well (Awaya, Miyake, Imaizumi, Shiose, Kanda, \& Komuro, 1973; von Noorden \& Maumenee, 1968). Recently, however, McCourt and Jacobs (1980), working with California ground squirrels, indicated that the effects of exposure to a chromatically restrictive environment may be reversible.

When adults are similarly placed in such restrictive environments, their visual systems, rather than losing sensitivity to the missing stimuli, may become somewhat desensitized to the stimuli predominating in that environment and may perhaps even become somewhat supersensitized to the stimuli missing in that environment, that is, to the stimuli of which they have been deprived (DeValois, 1977). The fully developed visual system has the ability to adapt to its ambient environment.

Several investigators have studied the effects, upon

We wish to express our thanks to $\mathrm{C}$. Stromeyer and $\mathrm{K}$. White for suggestions and helpful discussion, to Colonel D. Glick of Fort Rucker, Alabama, for lending us the anomaloscope, and to $\mathrm{D}$. Hood and $\mathbf{M}$. Powers for comments after a talk delivered at the Optical Society of America annual meeting. This research was supported in part by a National Institutes of Health Training Grant EY07046 and Research Grant EY03674 to Jay M. Enoch. Requests for reprints should be directed to the first author. both human adults (Hill \& Stevenson, 1976; Kohler, 1962; McCollough, 1965) and adult monkeys (LeGros Clark, 1942), of long-term exposure to restrictive chromatic environments, but, to our knowledge, none have quantitatively measured its effects upon sensitivity of various mechanisms of human color vision. Changes in color appearance in photopic environments have been noted (Hill \& Stevenson, 1976; Kohler, 1962).

The present study investigated the effects of a 1week exposure to the natural environment with one eye unoccluded and the other eye viewing the world through a red-colored filter. We found that, for the eye that had worn the red filter, (1) sensitivity to red stimuli decreased by a factor of about 2 , as measured up to $2 \mathrm{~h}$ after removal of the red filter, (2) unique yellow shifted to longer wavelengths after the removal of the filter, and (3) suprathreshold scotopic stimuli appeared distinctly bluish. Furthermore, we showed that, in general, it was the long-term exposure, per se, to a long-wave world that caused these changes. Recovery seemed complete within several days at most.

\section{METHOD}

Subjects

Three paid, emmetropic subjects-S.N., 22 years, male; N.M., 18 years, female; and C.W., 25 years, female-were employed.

\section{Apparatus}

The apparatus was the two-channel Maxwellian view visual testing device described in detail in Enoch and Hope (1972).

\section{Calibration}

A Pritchard Spectra-Physics photometer was employed in calculating retinal illuminance. We used a Beckman Acta III spectrometer to measure the transmittance of the red filter and the green Baird Atomic interference filter. The values of all other colored filters were obtained from the manufacturer's specifications.

\section{Procedure}

Red filter. After collecting baseline data over a number of independent sessions (as described below), the subjects wore a red filter over one eye on two separate occasions: once for a period of 6 days and a portion of the 7 th day, on which they were tested, 
and once, immediately upon awakening, either several weeks after the week-long stint (Observers N.M. and S.N.) or else immediately before it (C.W.), for only the duration of the 7 th day of the weeklong stint's wear period. During the week-long stint, the subjects wore the red filter continuously during their waking hours, sometimes replacing it with a pair of red goggles for showering. They were instructed to wear a black patch or the red filter over that eye while sleeping. One subject (S.N.) disobeyed this particular instruction. All subjects were instructed to be extremely careful to continuously exclude stray light from the eye.

The spectral characteristics of the red filter are shown in Figure 1. They are similar to those of a Kodak Wratten 29 filter. Both normal daylight and indoor lighting passing through the red filter were metameric, with spectral lights of roughly $630 \mathrm{~nm}$. The filter may be more properly described as orange-red; in fact, one observer (C.W.) described the bright sky as looking rather orangealmost yellow-through this filter. The filter was constructed by punching a hole in an opaque Bernell black eye occluder and inserting a correspondence piece of Laser protective goggle in the hole. Care was taken to eliminate stray light with black electrical tape, both around the edge of the hole and the edge of the eyepatch itself. It was verified that the subject retained at least 25 deg of visual field in any direction from the fovea when the eyes were fixed on a point $1 \mathrm{~m}$ straight ahead. The red filter presumably has very great density at short and middle wavelengths (beyond the resolution of the Beckman spectrometer), since it is made for Laser protection.

After removal of the red filter, each subject sat in the dark for $30 \mathrm{~min}$ before testing began. For interocular comparisons, data for the control eye were sandwiched between two sets of data for the experimental eye. At no time was data collected while the subject wore the red filter.

Thresholds. Thresholds for various color mechanisms and the scotopic mechanism were obtained at several retinal loci. For foveally presented stimuli, the subjects set increment thresholds by decreasing the radiance of the test until the flashing test stimulus (150 msec duration at a rate of $1 \mathrm{~Hz}$ ) was judged to have just disappeared. For stimuli presented more peripherally -5 or $10 \mathrm{deg}$ from the fovea-the subject fixated a dim red fixation spot and increased the radiance of the test until the flash was first seen. ${ }^{1}$ The test stimuli were 1-deg disks. For some conditions, a background field subtending 4 deg 24 min centered on the test field was continuously present as well. The aperture stops of both the background and the test beams were focused sharply in the center of the observer's entrance pupil, where they subtended $.3 \mathrm{~mm}$ in diameter.

Thresholds were measured for scotopic vision after the subject dark-adapted for $25 \mathrm{~min}$ after having been in an indoor environment for at least $1 / 2 \mathrm{~h}$. The test beam passed through a bluish

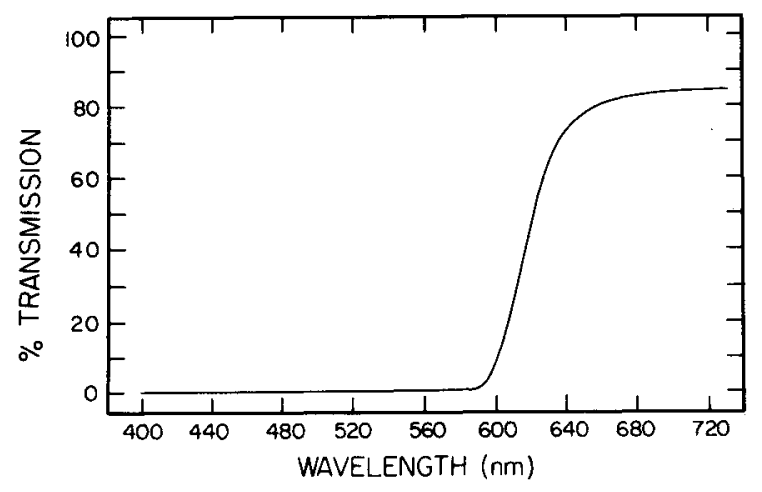

Figure 1. Spectral transmission of red filter.
Wratten 98 filter. The same Wratten 98 filter (which transmitted light virtually metameric with a spectral light of $452 \mathrm{~nm}$ ) was used to measure blue cone sensitivity upon a background light of 200 photopic trolands (Td) virtually metameric with a spectral light of $594 \mathrm{~nm}$ (Wratten 21). The blue cone thresholds were verified to lie on a plateau portion of the tvi curve, and were almost certainly mediated by $\pi_{1}$, but detection via $\pi_{2}$ was not unequivocally ruled out. A spectral test of $528 \mathrm{~nm}$ was used to presumably measure the sensitivity of $\pi_{4}$, both at absolute threshold and upon a deep red backgrond of $25 \mathrm{Td}$. Stiles-Crawford measurements, as well as subjective reports of color appearance, were used to ensure that cones were, in fact, mediating thresholds. Lastly, the sensitivity of $\pi_{5}$ at absolute threshold was measured using a test metameric with a spectral light of $678 \mathrm{~nm}$ (Wratten 70).

The different stimulus conditions were generally presented in the same order across sessions for foveal stimuli. The subjects always sat in the dark for $15 \mathrm{~min}$ before beginning testing. When comparing sensitivity between eyes, the order of presentation was alternated daily for one observer (C.W.) and determined haphazardly for another (N.M.). A third observer was tested almost exclusively monocularly. The data presented are the means of four settings.

At least $2 \mathrm{~min}$ in the dark was always taken between measurements of sensitivity using different stimulus conditions (including unique yellow-see below), and there was always an interval of at least 5-10 min for sets of thresholds measured through a given eye. The subjects adapted for about 2 min to the 25-Td deep red background field used in order to isolate $\pi_{4}$ and at least $3 \mathrm{~min}$ to the 200-Td yellow background field used to isolate the blue cone mechanism. The order of presentation for foveal stimuli was: (1) unique yellow, (2) green test on red background, (3) green test at absolute threshold (for C.W.), (4) red test at absolute threshold, (5) short wavelength test on yellow background, and (6) anomaloscope. When thresholds in the periphery were obtained in the same session as foveal data, they were obtained after the above sequence.

Unique yellow. Measurements of a subject's unique yellow were obtained in two ways. The first way was used to infer changes in the unique yellow wavelength. The dimensions of the background field were reduced to precisely coincide with the test field's ( $1 \mathrm{deg}$ ). The "background" beam passed through a deep red Wratten 70 filter, and the (collimated) test beam passed through an interference filter with a peak transmittance at $528 \mathrm{~nm}$ and a half bandwidth of $11 \mathrm{~nm}$. Both fields were presented steadily. The subject, after sitting in the dark for $15 \mathrm{~min}$, adjusted the radiance of the test so that the resultant of the red and green admixture field appeared neither reddish nor greenish (i.e., unique yellow) and then stared at this field for $1 \mathrm{~min}$. The subject then proceeded to adjust the test radiance in order to make the resultant field appear just discriminably too red and then just discriminably too green, and back and forth again in rapid succession. The subject did this for a series of 10 oscillations which were averaged to obtain the unique yellow setting. The resultant fields were about $50 \mathrm{Td}$ retinal illuminance.

In order to measure absolute values, as opposed to changes, of unique yellow, the test field was occluded and the background field was reduced to $1 / 2 \mathrm{deg}$ using a $2-\mathrm{mm}$ field stop. A Schott interference wedge was manually inserted into the system at this field stop conjugate with the subject's retina. The resultant stimulus had a nominal bandwidth of $5 \mathrm{~nm}$. The subject then directed the experimenter to translate the interference wedge-sometimes one way, sometimes the other-until unique yellow was achieved. This was done four times and averaged. The fields were about 175 Td.

Anomaloscope. The subjects made five settings per session with each eye, using a method of adjustment on a Schmidt-Hensch Model 11 Nagel anomaloscope. Settings were obtained for each eye with counterbalancing similar to that used for unique yellow and threshold settings. 


\section{RESULTS}

\section{Quantitative}

A week of wearing a red filter over one eye decreased absolute sensitivity to a long-wavelength red test in that eye by up to .4 log unit, as seen in Figures $2-4$. The decreases in sensitivity to a longwavelength test after wearing the red filter for a week are evidenced by the dip from baseline for the solid circles bounded by the dashed lines. Note that $\mathrm{Ob}$ server N.M. twice wore the red filter for a week. Sensitivity to middle-wavelength green stimuli (Figures 2,3 ) and to scotopic stimuli (Figure 4) did not appear to be altered to any obvious extent, while sensitivity to short-wave stimuli (Figures 2-4) mediated by blue cones may have remained the same or perhaps have been enhanced for some observers. ${ }^{2}$ Changes can be measured by comparing thresholds between the two eyes for a given mechanism both before and after patching (Figures 2, 3; Observers N.M. and C.W.), as well as by comparing thresholds between different mechanisms within one eye (Figure 4; Observers N.M. and S.N.). The decline in sensitivity to red appears to be entirely monocular, since it appears to be the same when measured either way for the one observer (N.M.) tested in both ways.

The loss in sensitivity to a red test was measured about $2 \mathrm{~h}$ after removal of the filter for Observer S.N., $1 / 2 h$ after for C.W., ${ }^{3}$ and $11 / 2 h$ after for N.M. (each time). It is not clear how long the loss lasted, but the loss appeared to be reduced by .15 log units

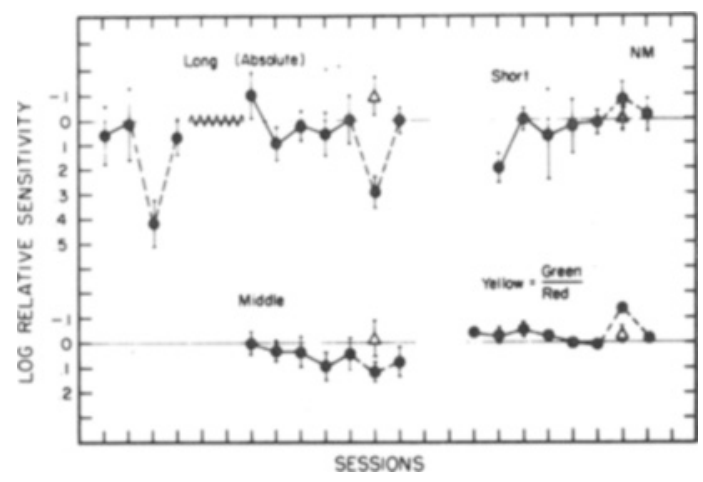

Figure 2. Daily interocular differences of log sensitivity (control eye/experimental eye) to long-, middle-, and short-wavelength tests and $\log$ radiance of green required to cancel red for unique yellow, all at the fovea. See Method section for details. Error bars represent one standard deviation (SD). "Absolute" refers to absolute sensitivity. Baseline data taken before wearing the red filter are to the left of the dashed lines. Data taken upon removal of the red filter after 1 week's wear is bounded by dashed lines. Triangles represent intraocular differences in sensitivity after wearing the filter for less than 1 day rather than 1 week. Data taken at least 1 day after removal of the filter is to the right of the dashed lines. Jagged line represents a gap of several weeks. Observer N.M.; experimental eye $=$ right eye.

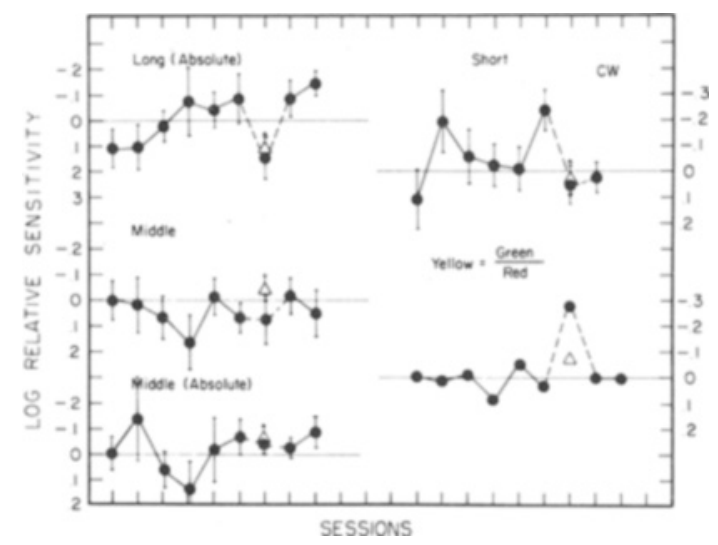

Figure 3. Same as Figure 2, except for Observer C.W.; experimental eye $=$ left eye.

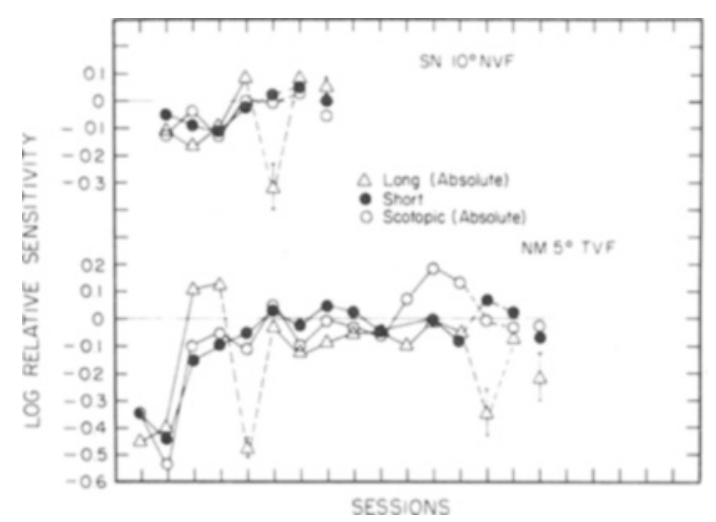

Figure 4. Parafoveal log photopic sensitivity to a long-wavelength test at absolute threshold ( $\Delta)$, to a short-wavelength test on plateau portion of tvi curve $(\cdot)$, and of $\log$ scotopic sensitivity at absolute threshold $(O)$. Baseline data and postfilter data are $a$ in Figure 2. Data offset to the right represent $\log$ sensitivity after wearing filter for less than 1 day rather than 1 week. Data have been translated vertically, so mean sensitivities for different mechanisms are equal (excluding data bounded by dashed lines which were obtained upon removal of the red filter). Sessions were not always run on consecutive days. Observers S.N. and N.M.; experimental eye $=$ right eye for S.N. Error ban ( \pm 1 SD) are drawn for eencitivity to long-wavelength teats after removal of the red fiter. SDs for remaining long-wavelength teats are generally about .05 log unlts.

by $1 \mathrm{~h}$ after removal of the filter for C.W. and by $.2 \log$ units $3 \mathrm{~h}$ after for N.M.

Two observers were tested the day after removal of the filter and showed complete recovery (rightmost point connected by dashed line in Figures $2-4$ ). The third (S.N.) was not tested until 13 days later, when he too had returned entirely to normal.

Unique yellow shifted to longer wavelengths as a function of wearing the red filter for a week. That is, less green was required to cancel a fixed red stimulus. (Equivalently, Figures 2 and 3 show that relatively more green was required for cancellation in the con- 
trol eye). Assuming that the red/green cancellation for unique yellow is linear (Larimer, Krantz, \& Cicerone, 1974; Nagy, 1979), finding the approximate value of the prefiltering unique yellow by using the interference wedge enables one to precisely deduce the shift of unique yellow in nanometers by using the data for red/green cancellation along with the assumption that each observer has red and green cone spectral sensitivities, as estimated by Smith and Pokorny (1975) using a pigment loss explanation for dichromacy. Observer C.W.'s unique yellow could be deduced to have shifted $12 \mathrm{~nm}$ from about $587 \mathrm{~nm}$, and N.M.'s could be deduced to have shifted $6 \mathrm{~nm}$ from about $580 \mathrm{~nm}$ (the second time she wore the red filter). Observer S.N.'s unique yellow shifted from $576 \pm 3 \mathrm{~nm}$ to $604 \pm 4 \mathrm{~nm}$, as measured directly with the interference wedge.

Anomaloscope settings appeared to remain unaltered for the two observers tested (S.N. and C.W.). ${ }^{4}$

We wanted to get some idea of how long the red filter had to be worn in order to induce the changes in visual sensitivity we recorded. Therefore, in order to decide whether the measured changes were due to the last day's wearing of the filter only and whether the previous 6 days' wear was superfluous, the experiment was repeated, but without the first 6 days' wear. So, for instance, N.M. wore the filter to sleep the night before testing and continued wearing it for $45 \mathrm{~min}$ the following day before removing it prior to testing. Observer C.W. wore the filter for $7 \mathrm{~h}$ and S.N. wore it for $1 \mathrm{~h}$ and $45 \mathrm{~min}$. The results are shown as triangles in Figures 2 and 3 and as offset to the right in Figure 4 in comparison with the results for a week's filter wear. The changes from baseline are clearly absent or reduced with one exception (sensitivity to red for C.W., for whom the sensitivity to a red test was measured at $1 \mathrm{~h}$ rather than at $30 \mathrm{~min}^{3}$ as with a week's filter wear). These results imply that the changes generally depend upon wearing the red filter for a relatively extended period of time.

\section{Subjective}

Following nondirective questioning techniques, all three subjects remarked after removal of the red filter that natural scotopic stimuli (as opposed to the 1-deg test flash at threshold) looked especially bluish": in the words of one (C.W.), "a lustrey [sic] bluethat of a bright blue sky." In the laboratory, conditions were almost certainly scotopic and not mesopic after removal of the red filter, since stimuli seen with the (relatively more dark-adapted) eye from which the filter had been removed 10 min previously were invisible to the subject's other eye as well as to the experimenter, and so could not have been detected with cones, which dark-adapt within several minutes. Observer $C$.W. reported that nighttime stimuli were still somewhat bluish $17 \mathrm{~h}$ later, while S.N. reported that the effect was still present 2 nights later. Observer
C.W. reported as well that deliberate blinking evoked a subjectively bright red flash under scotopic viewing conditions, both in the laboratory setting and elsewhere. Observer N.M. reported no differences between the two eyes after leaving the laboratory. Observer S.N. claimed that daytime stimuli appeared bluer through the experimental eye than though the control eye for as long as 5 days after removal of the red filter.

\section{DISCUSSION}

The results demonstrate the existence of an adaptational process which (1) requires a long-term exposure to long-wave stimuli of at least many hours and, in all likelihood, days in order to attain its measured strength and (2) is prolonged-lasting at least several hours and sometimes for more than 1 day.

The changes in sensitivity cannot be ascribed to preneural changes in the eye, since anomaloscope settings appeared to remain unaltered and Stiles-Crawford measurements made at fixation and elsewhere (obtained as in Enoch \& Hope, 1972) either changed nonsignificantly or not at all. Furthermore, if the optics of the eye suffered, the eye would have been expected to lose sensitivity to middle-wavelength tests, as it did to long-wavelength tests. But it did not, leaving only an implausible cancellation of preretinal and more proximal factors as a viable alternative. The changes can therefore be assumed to be neural.

The adaptational process most likely involves postreceptoral changes in sensitivity. It is conceivable that receptor crosstalk, via horizontal cells, say, or directly between receptors, could induce adaptational changes within a given class of cones. That is, the adaptation itself could be intrareceptoral, even though its cause must be inter- or postreceptoral. However, equating all the adaptation with a sensitivity scaling factor within individual red cones would lead to a contradiction, since the shift of the unique yellow wavelength and the loss in long-wavelength sensitivity would then be quantitatively mutually incompatible. For instance, if all the adaptation served to merely alter the relative sensitivities of the different cone types by a constant factor, then Subject N.M.'s .3-logunit loss in sensitivity to red tests would imply a unique yellow shift of over $20 \mathrm{~nm}$ rather than $6 \mathrm{~nm}$ as was observed, granted no change in green cone sensitivity. In fact, if there were any change in green cone sensitivity at all, the data (Figure 2 ) suggest it would be a decrease, which would serve to further widen the discrepancy between the unique yellow shift and decrease in long-wavelength sensitivity. It must be noted that equating the adaptation within cones with a sensitivity scaling factor and not, say, a compressive nonlinearity is open to question (Hood, 1978), especially since unique yellow was measured with continuous viewing, while sensitivity to long- 
wavelength tests was measured with 150 -msec flashes. But such an assumption is probably sufficiently near the truth so as to be applicable, at least to the extent that color appearance is determined by transients at the border of the field (Krauskopf, 1963). The differences between observers-a relatively large decline in long-wavelength sensitivity coupled with a small shift in unique yellow for N.M. vs. a relatively smaller decline in long-wavelength sensitivity coupled with a larger shift in unique yellow for C.W.--suggest that adaptation within the red cones alone cannot be entirely responsible for the objectively measured charges produced by wearing the red filter. Furthermore, the strikingly colored appearance of scotopic stimuli makes it reasonably certain that there is postreceptoral adaptation, although subjective data must, of course, be treated with caution.

In conclusion, the weight of the evidence makes it quite likely that wearing a red filter over one eye for a week induces postreceptoral adaptation in monocular pathways. Receptor-level adaptation as well cannot be ruled out.

It is difficult to say where the site or sites of adaptation may be. Empirically, we found that the unique yellow shift was not monotonic across observers with the decrease in sensitivity to red. This suggests that there is more than one site of adaptation but does not prove it since, if, for instance, different observers have different populations of opponent color cells, it is plausible that a given loss of sensitivity to longwavelength tests may be associated with different (changes in) color opponent balance points for these different observers. It is known that color opponent cells may exhibit rod input (Wiesel \& Hubel, 1966), and so models incorporating sensitivity changes at some color opponent level are compatible with the subjectively colored appearance of scotopic stimuli.

Much work using quite varied approaches has been published during the past several years and has shown the importance of second-site postreceptoral mechanisms of chromatic adaptation (cf. Benzchawel \& Guth, in press; Eisner \& MacLeod, 1981; Finkelstein \& Hood, 1981; Jameson, Hurvich, \& Varner, 1979; Pugh \& Mollon, 1979; Stabell \& Stabell, 1975; Stromeyer \& Sternheim, 1981; Valeton $\&$ van Norren, 1979; Wandell \& Pugh, 1980). Stabell and Stabell found scotopic color contrast effects after short-term (10-sec) chromatic adaptation, although they did not explicitly discuss the locus of the effects, and Jameson et al. found changes in unique yellow lasting several minutes which they ascribed to postreceptoral opponent color processes after intermittently (10 sec on, $10 \mathrm{sec}$ off) adapting the eye to colored stimuli for $5 \mathrm{~min}$. It is quite possible that the Stabell and Stabell and Jameson et al. findings are the short-term equivalent of ours. Stromeyer and Sternheim (1981) found that adding a green background to low-frequency red gratings superimposed on bright red adapting fields improved the visibility of the gratings, whereas adding red backgrounds did not improve the visibility of green gratings on green adapting fields. It is likely that the extent of opponent-level polarization is important in both Stromeyer and Sternheim's and our findings. In this context, it would be interesting to repeat our experiment with a green rather than a red filter, as well as to repeat the red filter experiment looking at the effect of colored backgrounds upon sensitivity to long-wavelength tests after removing the red filter. The general conclusion that studies on postreceptoral chromatic adaptation seem to reach is that the degree of opponent level polarization greatly affects visual sensitivity; for instance, a second site's response may saturate, making it insensitive to any incremental stimuli which would further polarize it. Perhaps wearing a red filter for a week can cause a relatively prolonged polarization at a second site in the red direction which may serve to decrease sensitivity to long-wavelength tests, shift unique yellow (the red/ green balance point) to a longer wavelength, and make scotopic stimuli take on a contrast color.

Finally, it is probable that exposure to light greatly accelerated the return to normal sensitivity after removing the red filter. That is, it seems likely that had the observers not slept the night before removal of the filter but, instead, had functioned in a light environment during that time without wearing the red filter, then the changes would have been appreciably smaller than those actually measured. Since the first 6 days' wear of the red filter was not superfluous, in some sense the visual system must have remained in a state of long-wave readiness during the time spent sleeping. It therefore seems probable that the visual system has some inertial tendency toward an equilibrium upon which local adaptational changes may be superimposed, which may be altered by longterm changes in the environment.

\section{REFERENCES}

Awaya, S., Miyake, Y., Imaizumi, Y., Shiose, Y., Kanda, T., \& Komuro, K. Amblyopia in man suggestive of stimulus deprivation amblyopia. Japanese Journal of Ophthalmology, 1973, 17, 69-82.

Baker, F. H., Grigg, P., \& von Noorden, G. K. Effects of visual deprivation and strabismus on the response of neurons in the visual cortex of the monkey including studies on the striate and prestriate cortex in the normal animal. Brain Research, 1974, 66, 185-208.

Barlow, H. B. Visual experience and cortical development. Nature, 1975, 258, 199-204.

Benzchawez, T., \& GUth, S. L. Post receptor chromatic mechanisms revealed by flickering vs. fused adaptation. Vision Research, in press.

Blakemore, C., \& Cooper, G. F. Development of the brain depends on the visual environment. Nature, 1970, 228, 477-478. 
DeVALors, K. K. Spatial frequency adaptation can enhance contrast sensitivity. Vision Research, 1977, 17, 1057-1065.

Eisner, A., \& MacLeod, D. I. A. Flicker photometric study of chromatic adaptation: Selective suppression of cone inputs by colored backgrounds. Journal of the Optical Society of America, $1981,71,705-718$.

ENoch, J. M., \& HoPE, G. M. An analysis of retinal receptor orientation: III. Results of initial psychophysical tests. Investigative Ophthalmology, 1972, 11, 765-782.

Finkelstein, M. A., \& Hoon, D. C. Cone system saturationMore than one stage of sensitivity loss. Vision Research, 1981, 21, 319-328.

Hill, A. R., \& Stevenson, R. W. W. Long term adaptation to ophthalmic tinted lenses. Modern Problems in Ophthalmology (Color Vision Deficiencies III), 1976, 17, 264-271.

Hirsch, H. V. B., \& SpinelLI, D. N. Visual experience modifies distribution of horizontally and vertically oriented receptive fields in cats. Science, 1970, 168, 869-871.

Hood, D. C. Psychophysical and physiological tests of proposed physiological mechanisms of light adaptation. In J. Armington, J. Krauskopf, \& B. Wooten (Eds.), Visual psychophysics: Its physiological basis. New York: Academic Press, 1978.

Jameson, D., Hurvich, L. M., \& Varner, F. D. Receptoral and postreceptoral processes in recovery from chromatic adaptation. Proceedings of the National Academy of Sciences, 1979, 76, 3034-3038.

KoHLE R, I. Experiments with goggles. Scientific American, 1962, 206(5), 62-72.

KRAUSKOPF, J. Effect of retinal image stabilization on the appearance of heterochromatic targets. Journal of the Optical Society of America, 1963, 53, 741-744.

Kuffler, S. W., \& Nicholls, J. G. From neuron to brain. Sunderland, Mass: Sinauer, 1976.

Larimer, J., Krantz, D. H., \& Cicerone, C. M. Opponentprocess additivity I: Red/green equilibria. Vision Research, 1974, 14, 1127-1140.

LeGros Clark, W. E. The Doyne Memorial Lecture: The anatomy of cortical vision. Transactions of the Ophthalmological Society UK, 1942, 62, 229-245.

McCollovgh, C. The conditioning of color perception. American Journal of Psychology, 1965, 78, 362-378.

McCounT, M. E., \& JAcoBs, G. H. Rearing ground squirrels under spectrally restricted conditions reduces the proportion of spectrally opponent units in the optic nerve. Investigative Ophthalmology and Visual Science Supplement, 1980, $19,7$.

NAGY, A. L. Unique hues are not invariant with brief stimulus duration. Vision Research, 1979, 19, 1427-1432.

Pugh, E. N., \& Mollon, J. D. A theory of the $\pi_{1}$ and $\pi_{3}$ color mechanisms of Stiles. Vision Research, 1979, 19, 293-312.

Smith, V. C., \& Pokonny, J. Spectral sensitivity of the foveal cone photopigments between 400 and $500 \mathrm{~nm}$. Vision Research, 1975, 15, 161-171.

Stabell, U., \& Stabell, B. Scotopic contrast hues triggered by rod activity. Vision Research, 1975, 15, 1115-1118.

Stromeyer, C. F., \& Sternheim, C. E. Visibility of red and green spatial patterns upon spectrally mixed adapting fields. Vision Research, 1981, 21, 397-407.

Valeton, J. M., \& VAN NorRen, D. Transient tritanopa at the level of the ERG b wave. Vision Research, 1979, 19, 689-693.

von Noorden, G. K., \& Maumenee, E. Clinical observations on stimulus deprivation amblyopia (amblyopia ex anopsia). American Journal of Ophthalmology, 1968, 65, 220-224.

Wandell, B. A., \& PUGH, E. N. Detection of long-duration long-wavelength incremental flashes by a chromatically coded pathway. Vision Research, 1980, 20, 625-636.

Wiesel, T. N., \& Hubel, D. H. Spatial and chromatic interactions in the lateral geniculate body of the rhesus monkey. Journal of Neurophysiology, 1966, 29, 1115-1156.

WiEsel, T. N., \& HubEL, D. H. Ordered arrangement of orientation columns in monkeys lacking visual experience. Journal of Comparative Neurology, 1974, 158, 307-318.

\section{NOTES}

1. Since we are concerned with measuring changes of sensitivity (both interocularly, using a method of descent, and intraocularly, using a method of ascent) rather than absolute levels of sensitivity, the use of two methods of obtaining thresholds is not unwarranted. A method of descent was used foveally in order to ensure fixation without the use of fixation spots.

2. A fourth (pilot) observer, wearing a pair of goggles with a red filter over one eye and an orange filter over the other for a week, seemed to show a roughly .4 log unit increase in blue cone sensitivity in the eye behind the red filter (the only one tested) at 15 deg nasal visual field.

3. The usual order of stimulus presentation was altered somewhat for this observer after removal of the red filter. Everything was the same as described in the Method section except that $\pi_{3}$ sensitivity was measured first. There was then 15 min more of dark adaptation, followed by the usual sequence.

4. However, a pilot observer who was tested only in the eye that had worn the red filter seemed to show a deutan shift. Without using the other eye as a control, it is not clear whether or not this is physiologically significant.

5. Similar scotopic color contrast effects have been noted previously after brief exposures $(10 \mathrm{sec})$ to photopic stimuli (Stabell \& Stabell, 1975).

(Manuscript received January 9, 1981; revision accepted for publication October 23, 1981.) 